\title{
A Stochastic Programming Approach on Aircraft Recovery Problem
}

\author{
Bo Zhu, Jin-fu Zhu, and Qiang Gao \\ College of Civil Aviation, Nanjing University of Aeronautics and Astronautics, Nanjing 211100, China \\ Correspondence should be addressed to Bo Zhu; acoustic.zhu@gmail.com
}

Received 12 May 2015; Revised 16 August 2015; Accepted 23 August 2015

Academic Editor: Yuanchang Xie

Copyright ( 92015 Bo Zhu et al. This is an open access article distributed under the Creative Commons Attribution License, which permits unrestricted use, distribution, and reproduction in any medium, provided the original work is properly cited.

\begin{abstract}
The unexpected aircraft failure is one of the main disruption factors that cause flight irregularity. The aircraft schedule recovery is a challenging problem in both industrial and academic fields, especially when aircraft restoration time is uncertain, which is often ignored in previous research. This paper established a two-stage stochastic recovery model to deal with the problem. The first stage model was a resource assignment model on aircraft schedule recovery, with the objective function of minimizing delay and cancellation cost. The second stage model used simple retiming strategy to adjust the aircraft routings obtained in the first stage, with the objective function of minimizing the expected cost on recourse decision. Based on different scenarios of restoration time, the second stage model can be degenerated as several linear models. A stochastic Greedy Simulated Annealing algorithm was designed to solve the model. The computational results indicate that the proposed stochastic model and algorithm can effectively improve the feasibility of the recovery solutions, and the analysis of value of stochastic solution shows that the stochastic model is worthy of implementation in real life.
\end{abstract}

\section{Introduction}

In dynamic operation circumstances, airlines flight schedules will face different kinds of inevitable stochastic disruptions and will deviate from regular operations. As the development of air transportation, the flight schedule is planned pretty tight and the disruption often propagates in the flight network. Flight irregularity is a serious and widespread problem all over the world, which imposes significant cost to airlines, passengers, and the society. In 2013, the average on-time ratio was $78.4 \%$ in the U.S. according to the 16 main carriers' data from BTS. Each irregular flight will bring around $\$ 16,600$ loss on average, including expenses for fuel, maintenance, crew, the passenger time loss, and estimate of welfare loss. In China, the average on-time ratio was only $72.34 \%$ in 2013. The average delay time increased to almost 60 minutes, and around 2,100 irregular flights were handled per day. $40 \%$ of irregular flights are caused by airlines themselves, which is the most compared to other disruption factors in China. Aircraft breakdown, schedule temporary change, passenger issues, and so forth will hinder the flights operation regularly, numerous flights will be disrupted, and thousands of passenger itineraries will be destroyed. Aircraft are the most treasured resources for airlines; it is significant for dispatchers to retime the flight schedule and reassign aircraft and crews to recover the flight schedule as soon as possible.

The research on flight recovery problem has more than 60 years history, among which the aircraft recovery problem (ARP) is one of the most concerned. Teodorović and Guberinić studied how to recover the flight schedule to minimize the total passengers delay when unexpected aircraft failure happened. They used the branch and bound algorithm to solve some small scale examples [1]. Argüello et al. discussed the flight schedule recovery problem with temporary shortage of aircraft and applied GRASP algorithmic framework to rearrange aircraft routings [2]. Rosenberger et al. studied the aircraft schedule recovery problem under shortage of aircraft or change in airport capacity. They designed heuristic algorithm framework to solve the model [3]. Bratu and Barnhart studied the flight delay and cancellation decision considering the passenger arrival delay cost [4]. Tang et al. revised the GRASP method and designed Greedy Simulated 
Annealing (GSA) method to solve the recovery model [5]. Eggenberg et al. developed a column generation scheme to solve ARP [6]. Petersen et al. are known as the first scholars that studied the full integrated recovery formulation and approach with computational results presented [7]. Le and $\mathrm{Wu}$ presented iterative tree growing with node combination method to solve aircraft and crew recovery simultaneously [8]. Chan et al. established a model that integrates aircraft and passenger recovery, but no solution was offered [9]. Sinclair et al. designed a large neighborhood search heuristic algorithm to solve the integrated recovery of aircraft and passenger [10]. Hu et al. solved the integrated recovery problem of aircraft and passenger based on reduced timeband network and passenger transiting relationship [11]. Although some theoretical researches show good results in computational tests, they can barely be implemented well in real world because of the following reasons. Firstly, the disruptions are simply assumed as deterministic. For example, the restoration time of aircraft is assumed to be known as constant before decision making, which is usually hard to predict precisely even for the sophisticated maintenance staff. Secondly, as in dynamic circumstances, the recovery solution from deterministic model may be lack of robustness in operation. When the random variables become realized as time passes, the previous recovery plan may be infeasible or not satisfactory. Thus, it is necessary to study the stochastic model and algorithm on the problem. There are some researches on uncertain theory in air transportation field, such as design and optimization on flight network [12] and the flight scheduling problem [13-15]. In airline operation area, Rosenberger et al. worked on the simulation software that controls the uncertain delay time [16]. Mou and Zhao built an uncertain programming model with chance constraint and solved it based on classic Hungarian algorithm to deal with the recovery problem under stochastic flight time [17]. Arias et al. proposed a combined methodology using simulation and optimization techniques to cope with the stochastic aircraft recovery problem [18].

In this paper, we developed a two-stage stochastic model to formulate the stochastic ARP and designed a stochastic algorithm based on GSA to solve the model. As far as we know, this paper is the first to bring the uncertain aircraft restoration time into the recovery problem.

\section{Problem Statement and Model}

When aircraft failure happens, there are several strategies to recover the flight schedule back to the regular status. The basic strategies to recover the flight timetable are delay and cancellation. For aircraft rerouting problem, strategies such as aircraft swap, type substitution, reserved aircraft, and ferry can be used. In Figure 1, a small example of aircraft routings is illustrated. The grey area means aircraft A2 found failure at 07:30, and the anticipated recovery time will be 13:15. The Airline Operation Control Center (AOCC) can choose strategy to cancel flights 4 and 5; or they can just delay flights 4-7 in a row; or aircraft A1 and A2 can switch routings at 08:00 and so forth. The figure shows a classic deterministic aircraft schedule recovery problem, and all the rescheduled plans are generated on the premise that the recovery time of A2 is known in advance.

However, the recovery time above is an expected value which is usually given by airline maintenance staff. The value barely equals the actual one, which may make the current recovery plan not satisfactory or even infeasible. For example, if at 07:30 AOCC chooses to delay flight 4 until 13:15, but when it comes to the time 13:15, aircraft A2 is not available to use yet, more delays or cancellations will be incurred. Anther situation is that A2 is ready for use earlier than 13:15; then, a more cost-saving plan might be optional. Since the new disruption information will be updated frequently, it will be time consuming to redo the whole optimization iteratively. An intuitive thought is to generate a robust recovery plan and when the random restoration time of aircraft is determined, it is still feasible and satisfactory with simple recourse decision.

In this paper, the concept of stochastic aircraft recovery time is introduced, and a two-stage aircraft schedule recovery model is established. The classic two-stage stochastic fixed recourse linear model is proposed by Dantzig [19] and Beale [20]. The model is designed to choose one decision, which makes the cost of current decision and the expectation of future recourse cost minimized [21]. For flight recovery problem, the two-stage model can evaluate the influences of different rescheduled plans and the uncertainty of the disruption factors, thereby making robust decisions. In our model, the first stage model is the deterministic resource assignment model of ARP. Based on different stochastic scenarios of aircraft recovery time, the recourse model will adjust the recovery plan obtained in the first stage. The strategy of recourse model is retiming the flights but maintaining aircraft routings generated in the first stage. It ensures the feasibility of recourse model and the simple linear formulation can guarantee the computational speed. Cancellation and aircraft swap can also be implemented as strategies in recourse model, but they will not change the essence of the model.

2.1. Stochastic Model. In the research of deterministic aircraft recovery problem, resource assignment model is one of the most prevalent ones because it can describe the problem in a complete and concise way. Our first stage model is referred to Argüello et al.s model [2]. Flights are implicitly generated as routings which will be assigned to aircraft. The notions are defined as follows:

(1) Sets are as follows:

$F$ : flight set, indexed by $i$.

$K$ : available aircraft set, indexed by $k$.

$A$ : airport set, indexed by $a$.

$P$ : feasible aircraft routing set, indexed by $j$.

(2) Parameters are as follows:

$a_{i, j}$ : equal to 1 if flight $i$ is in aircraft routing $j$, otherwise, equal to 0 .

$b_{j, a}$ : equal to 1 if aircraft routing $j$ will end at airport $a$, otherwise, equal to 0 . 


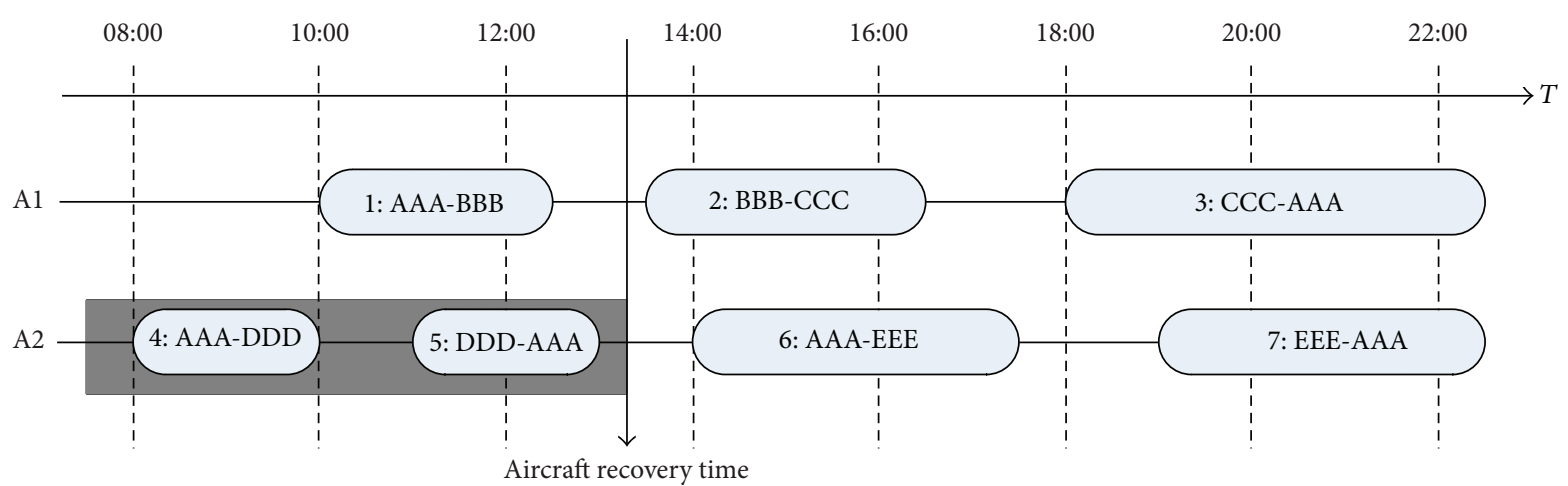

FIGURE 1: A small case of aircraft routings.

$c_{i}$ : the cancellation cost of flight $i$.

$h_{a}$ : the required amount of aircraft at airport $a$, at the end of recovery process.

$d_{j}^{k}$ : the delay cost of assigning aircraft $k$ to routing $j$.

(3) Decision variables are as follows:

$x_{j}^{k}$ : equal to 1 if aircraft $k$ is assigned to routing $j$, otherwise, equal to 0 .

$y_{i}$ : equal to 1 if flight $i$ is cancelled, otherwise, equal to 0 .

Using the above notations, the first stage resource assignment model for aircraft recovery problem is

$$
\begin{array}{ll}
\min & Z=\sum_{k \in K} \sum_{j \in P} d_{j}^{k} x_{j}^{k}+\sum_{i \in F} c_{i} y_{i} \\
\text { s.t. } & \sum_{k \in K} \sum_{j \in P} a_{i, j} x_{j}^{k}+y_{i}=1, \quad \forall i \in F \\
& \sum_{k \in K} \sum_{j \in P} b_{j a} x_{j}^{k} \geq h_{a}, \quad \forall a \in A \\
& \sum_{j \in P} x_{j}^{k}=1, \quad \forall k \in K \\
& x_{j}^{k}=0,1, \quad \forall(j, k) \in P \times K \\
& y_{i}=0,1, \quad \forall i \in F .
\end{array}
$$

The objective function (1) minimizes the cost of flight delay and cancellation. Constraints (2) are flight coverage constraints. For any flight $i$, it either be cancelled or assigned to a routing. Constraints (3) are aircraft balance constraints, which require certain amount of aircraft in different airports at the end of recovery process to preserve the future regular operation. Constraints (4) confine that each aircraft can only be assigned to one routing. Constraints (5) and (6) are nonnegative constraints for decision variables.

The deterministic model has an underlying work: the aircraft routings are already generated on the premise that aircraft recovery time is fixed. However, as we mentioned above, it is hard to determine the time in real operation. The research on aircraft reliability and maintainability [22] also supports this point of view. Therefore, an expected cost that is incurred by stochasticity is added to the optimization model; it reflects the possible changes of the rescheduled plans in the first stage. The general stochastic model formulation is as follows:

$$
\begin{aligned}
\mathrm{RP}=\min W=\min & (Z+\mathbb{Q}(x, y)) \\
\text { s.t. } & \mathbf{A}[x, y]=\mathbf{b} \\
& x, y=0,1 .
\end{aligned}
$$

The objective function (7) of the stochastic model consists of two parts. The first one is the objective function (1); the second one $\mathbb{Q}(x, y)$ is the expected cost of the future recourse decision on the rescheduled plans obtained at the first stage. Here, and in the following text, $x$ and $y$ are the simplified symbols which denote $x_{j}^{k}$ and $y_{i}$ in the deterministic model, respectively. Formula (8) is the general form of constraints (2)-(4). Constraints (9) are the nonnegative constraints. It is a standard two-stage recourse stochastic integer programming model.

2.2. Recourse Model. Since operations of flight schedule weave so many resources together, frequent severe changes on recovery plan are not preferred. Thus, it is meaningful to get a flexible, robust but also cost-saving recovery plan when disruption happens. Particularly, as the time passes, when the uncertain variables are determined, the selected rescheduled plan in the first stage can be implemented smoothly with or without minor adjustments. To obtain such rescheduled plan quickly is more acceptable than simply the pursuit of optimal solution in deterministic model of NP-Hard problem.

Figure 2 illustrates one rescheduled plan obtained in the first stage model from the same example in Figure 1. It swaps aircraft routings of aircraft $\mathrm{A} 1$ and $\mathrm{A} 2$ and delayed the flights 1-3. Obviously, the plan is drawn on the given aircraft recovery time, which is the end of the grey interval. In reality, the A2 recovery time may be a random variable with 


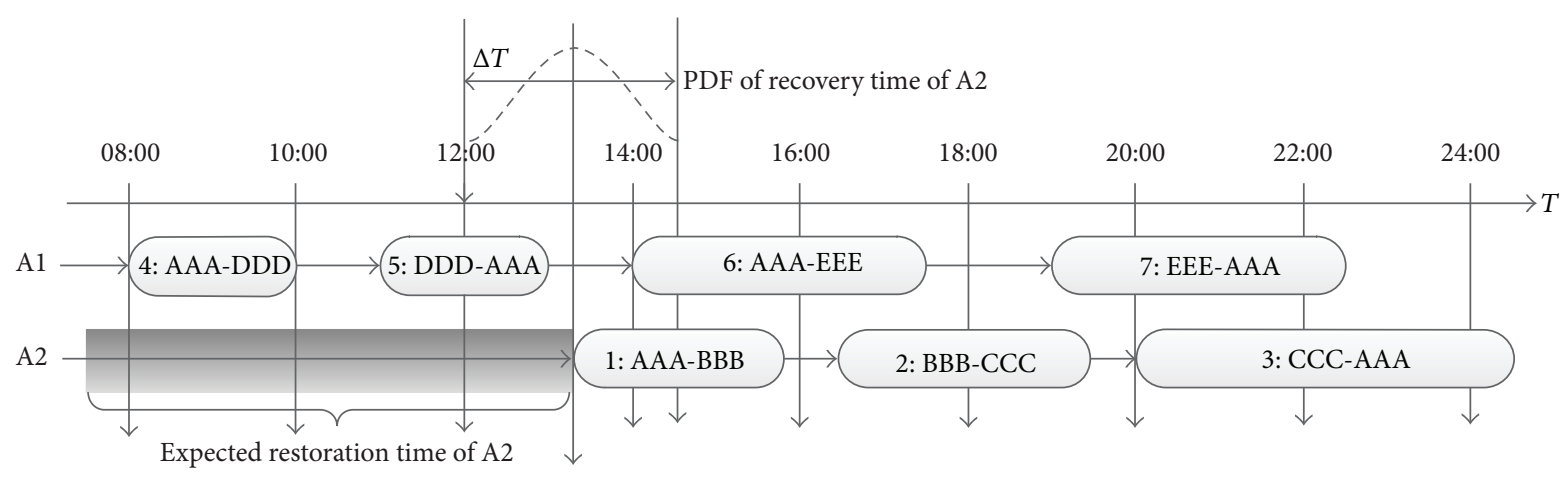

FIGURE 2: Illustration on recovery time of aircraft and recovery plan.

probability density function (PDF) curve in the figure, and its range is $\nabla T$. If A2 turns out to be ready at 14:00, then flights 13 will be redelayed in a row; if the new arrival time of flight 3 is beyond the curfew time of airport AAA, it will be cancelled, which will break the aircraft balance also; or it will be delayed until the curfew time is over, which will impose severe delay to the flight. This situation will be reflected in terms of risk cost in the recourse model.

The deterministic aircraft schedule recovery problem is an NP-hard problem; so no algorithm can be proved to be capable of obtaining optimality in polynomial time. A quick recovery solution is preferred and sometimes required. For two-stage stochastic model, there are a bunch of recourse models to be solved on each feasible solution obtained in the first stage. It requires the recourse model to be simple to solve. Let $\mathbf{T}$ denote the aircraft recovery time vector. It consists of every disrupted aircraft recovery time, which is considered as a continuous variable, and every available time for undisrupted aircraft which is a constant variable. Then, the objective function of the recourse model can be modeled as $\mathbb{Q}(x, y)=\int \mathbb{Q}(x, y, \mathbf{T}) \mathrm{d} \mathbf{T}$. Since the objective function is nonlinear and the PDF of the random variable is usually hard to obtain as well, we can discretize the aircraft recovery time without losing precision. The combinations of discretized points from every aircraft construct the finite scenario set $\Omega$. Let $\omega \in \Omega$ denote one scenario (combination), and $\operatorname{Pr}(\omega)$ is the probability of $\omega$. The recourse cost of the rescheduled plan can be expressed in the following:

$$
\mathbb{Q}(x, y)=\sum_{\omega} \mathbb{Q}(x, y, \omega) \operatorname{Pr}(\omega) .
$$

Besides notions in the first stage model, some other notions used in recourse model are listed as follows:

(1) Parameters are as follows:

$t_{i}$ : fly time of flight $i$.

$\rho_{i}$ : unit delay cost of flight $i$ (per minute).

$d_{i}^{\mathrm{s}}$ : original scheduled time of departure of flight $i$.

$d(i)$ : departure airport of flight $i$.

$r(i)$ : arrival airport of flight $i$.

$t_{a}$ : starting time of curfew on airport $a$. $\varphi_{i}$ : cost of breaking curfew regulation of flight $i$. $g_{k}$ : minimum turnaround time of aircraft $k$.

$t_{\omega}^{k}$ : recovery/ready time of aircraft $k$ under scenario $\omega$; so the random vector $\xi(\omega)=\left(t_{\omega}^{k}, k=\right.$ $1, \ldots,|K|)$.

$\Delta_{i}$ : delay time of flight $i$ obtained from optimization on the first stage.

$p(i)$ : predecessor flight of flight $i$ in the same aircraft routing after optimization on the first stage.

(2) Decision variables are as follows:

$d_{i, \omega}$ : new estimated time of departure of flight $i$ under scenario $\omega$.

$r_{i, \omega}$ : new estimated time of arrival of flight $i$ under scenario $\omega$.

$\Delta_{i, \omega}$ : estimated delay time of flight $i$ under scenario $\omega$.

$v_{i, \omega}$ : equal to 1 if flight $i$ violates the curfew requirement under scenario $\omega$, otherwise, equal to 0 .

The recourse model can be established as follows:

$$
\begin{array}{ll}
\min \quad \mathbb{Q} & =\sum_{\omega} \mathbb{Q}(x, y, \omega) \operatorname{Pr}(\omega) \\
& =\sum_{\omega}\left(\sum_{i} \rho_{i}\left(\Delta_{i \omega}-\Delta_{i}\right)+\varphi_{i} v_{i, \omega}\right) \operatorname{Pr}(\omega) \\
\text { s.t. } \quad & r_{i, \omega}=d_{i, \omega}+t_{i}, \quad \forall i \in F, \forall \omega \in \Omega \\
& d_{i, \omega}-d_{i}^{s}=\Delta_{i, \omega}, \quad \forall i \in F, \forall \omega \in \Omega \\
& d_{i, \omega} \geq t_{\omega}^{k} \sum_{j \in P} a_{i, j} x_{j}^{k}, \quad \forall(i, k) \in F \times K, \quad \forall \omega \in \Omega \\
& d_{i, \omega}-r_{p(i), \omega} \geq g_{k} \sum_{j \in P} a_{i, j} x_{j}^{k},
\end{array}
$$




$$
\begin{aligned}
& v_{i, \omega: d_{i, \omega} \geq t_{d(i)} \| r_{i, \omega} \geq t_{r(i)}} \geq 1, \quad \forall i \in F, \quad \forall \omega \in \Omega \\
& v_{i, \omega: d_{i, \omega}<t_{d(i)} \& r_{i, \omega}<t_{r(i)}} \leq 0, \quad \forall i \in F, \quad \forall \omega \in \Omega \\
& d_{i, \omega}, r_{i, \omega}, \Delta_{i, \omega} \geq 0, \quad \forall i \in F, \forall \omega \in \Omega \\
& v_{i, \omega}=0,1, \quad \forall i \in F, \forall \omega \in \Omega \text {. }
\end{aligned}
$$

The objective function of the recourse model (11) minimizes the expected cost on the recourse strategy of the first stage plan due to uncertainty of aircraft recovery time. For each scenario $\omega$, the recourse cost contains two parts: one is cost of flight retiming; if the aircraft can be ready before the expected time, $\Delta_{i \omega}-\Delta_{i}$ will be negative value; the other one is the risk cost of curfew breaking after retiming in the recourse stage. Constraints (12) are flight consistency constraints, which define the relationship between flight departure, arrival, and delay time. Constraints (13) require that aircraft cannot fly flights until it is ready. Constraints (14) require the minimum turnaround time (MTT) of adjacent flights in one aircraft routing. Notice that variables $t_{\omega}^{k}$ and $p(i)$ depend on the decision variables $x$ and $y$ in the first stage; once they are determined and passed to the recourse model, constraints (13) and (14) are degenerated to be linear constraints. Constraints (15) determine the value of $v_{i, \omega}$ for each flight $i$ under scenario $\omega$; if new departure/arrival time of $i$ violates the curfew time of its departure/arrival airport, $v_{i, \omega}$ is forced to be 1 ; otherwise, it should be 0 . Constraints (16) are the nonnegative constraints of the model.

\section{Algorithm}

Although solving the deterministic model of ARP is already very complicated; there are some successful results on the research of the algorithm. Precise algorithm such as column generation [6] and heuristic methods such as GRASP [2] and GSA [5] can obtain satisfactory solutions in tractable time. To extend the deterministic model to stochastic, one will make the scale of the problem larger and increase the computation complexity. If the recourse model is linear and the scenarios are limited, the stochastic model can always be transferred to equivalent deterministic model, which makes the problem scale even larger. Different from long-term scheduling problem, a quick solution is required for recovery problem. To solve the two-stage stochastic model efficiently, we design stochastic algorithm framework combining GSA [5] and simple retiming strategy, which concerns the stochastic structure of the problem.

For the first stage model, decision variables $x$ and $y$ can be obtained through GSA algorithm. As paper [5] states, the algorithm has 3 steps generally as follows:

(1) Construct initial feasible solution by delaying the disrupted aircraft routings.

(2) Generate neighboring solutions through 5 operations on disrupted pairs of aircraft routings. Disrupted pair of aircraft routings refers to two aircraft routings which include at least one disrupted aircraft in order to conserve the undisrupted aircraft routings. The 5 operations on each disrupted pair of aircraft routings are flight cycle insertion, flight string insertion to the routing tail, flight string swap, tail string swap, and flight cycle cancellation.

(3) Choose neighboring solutions from a Restricted Candidate List (RCL, which consists of cost-saving neighbors) or a Back Restricted Candidate List (BRCL, which consists of cost-increase neighbors) to substitute the original routings to get new solution.

Once the decision variables in the first stage model are passed to the second stage model, the model can be degenerated to several easy-solving linear optimization recourse models. As the aircraft routing's flights are fixed in the recourse model, the only adjustment of the rescheduled plan is to retime the flights as tight as possible (only consider aircraft recovery time and MTT) to minimize the objective function as long as it is feasible. If the retiming solution is not feasible, which can only be curfew violation under such condition, there are two ways to deal with this: one is to cancel the violation flight; the other one is to delay the flight until curfew is over. The cost of curfew breaking will be represented as $\varphi_{i}$. Therefore, $Q(x, y, \omega)$ will be obtained for each scenario $\omega \in \Omega$, and $\mathbb{Q}(x, y)$ can be computed according to (10) since scenarios are independent of each other.

The end criterion can be set as upper bound of the computational time since the recovery problem requires quick response in real operation. It can also be set as enough number of solutions or maximum iteration number, which will give the decision maker a lot of flexibility in operation. The detail algorithm steps are described as follows.

Step 1 (initialization). Let $l$ denote iteration number. $Z^{0}$ denotes the initial objective function value and $Z^{l}$ denotes the objective function value of the first stage model in the $l$ th iteration. $Q^{l *}(\omega)$ denotes the optimal value of the recourse model under scenario $\omega$ and $\mathbb{Q}^{l *}$ denotes the optimal value of the second stage model in $l$ th iteration. $W^{*}$ denotes the best objective function value of the stochastic model so far. $W^{l}$ denotes the current objective function value in $l$ th iteration of the stochastic model. Let $l=0, Z^{0}=Z^{l}=0, Q^{l *}(\omega)=$ $\mathbb{Q}^{l *}=0$, and $W^{*}=W^{l}=\infty$.

Step 2. Construct initial feasible solution in the first stage model. Take the expectation of aircraft recovery time as constant variable; then, delay the aircraft routings in a row to get $Z^{0}$. Set $l=1$ and $Z^{l}=Z^{0}$.

Step 3. Construct neighboring solutions through 5 different operations; choose the optimal neighboring solution for every pair of aircraft routings.

Step 4. Evaluate the neighboring solution. If the neighboring solution can decrease the objective function value of the first stage model, then add it to RCL; otherwise, add it to BRCL.

Step 5. If RCL is not empty, randomly select some neighboring solutions. If RCL is empty, randomly select neighboring solutions from BRCL, and determine whether to accept them 
according to Metropolis acceptance criterion from Simulated Annealing algorithm.

Step 6. Substitute the original aircraft routings using chosen neighboring solutions; obtain $Z^{l}$.

Step 7. Pass the values of decision variables of the current solution in first stage model to the recourse model, and degenerate the recourse model.

Step 8. For each disruption scenario $\omega$, solve the recourse model by retiming the stochastic aircraft routings, and obtain the optimal objective function value $\mathbb{Q}^{l *}(\omega)$.

Step 9. Compute $\mathbb{Q}^{l *}=\sum_{\omega} \mathbb{Q}^{l *}(\omega) P(\omega)$, and the total cost of the two-stage model $W^{l}=Z^{l}+\mathbb{Q}^{l *}$. If $W^{l}<W^{*}$, update $W^{*}=W^{l}$ and preserve the current recovery plan.

Step 10. Set $l=l+1$; if end criterion is met, the current plan is the best one so far; quit the algorithm, if not, go back to Step 3.

The flow chart of the algorithm is illustrated in Figure 3.

3.1. Algorithm Complexity. In the $l$ th iteration, for the first stage, an aircraft routing that consists of $p$ flights will need $O(p)$ time to delay the flights to obtain the initial feasible solution. For a pair of aircraft routings that consist of $p$ and $q$ flights, respectively, the time to construct neighboring solutions by 5 different operations is $O\left(p^{2} q^{2}\right)$. Suppose the total number of aircraft routings is $n ; m$ of them are disrupted due to aircraft breakdown; there will be $m(n-1)$ combinations of routing pairs, and the time of construct neighboring solutions in $l$ iterations is $O\left(\operatorname{lm}(n-1) p^{2} q^{2}\right)$. For one disruption scenario, the recourse model has $m$ disrupted aircraft routings; if they contain $r$ flights in each routing, then the computational time on the recourse model will be $O(m r)$. Suppose every disrupted aircraft has $\omega$ discrete recovery times; the scenarios of the whole problem will be $\omega^{m}$, and the objective function of recourse model needs $O\left(m r \omega^{m}\right)$ to compute. In real world operation, an aircraft cannot execute too many flights in one day; usually $p, q, r \leq 10$. To sum up, the algorithm time complexity will be $O\left(\ln m^{2} \omega^{m}\right)$. It is almost impossible that many aircraft have unexpected maintenance at the same time; so the value of $m$ cannot be very large; meanwhile, since there is no need to discretize the aircraft restoration time interval to get too many points as we mentioned before, the value of $\omega$ for each aircraft cannot be very large. Thus, in real operations, the computational time can be controlled and the algorithm can be regarded as quasilinear.

\section{Computational Test}

A case from a Chinese airline is studied in this section. Table 1 shows the original flight schedule snapshotted from the daily flight schedule. Std and Sta in the first row mean original scheduled time of departure and arrival, respectively.
TABLE 1: Original flight schedule.

\begin{tabular}{lccccc}
\hline Aircraft & Flight & Dep. & Arr. & Std. & Sta. \\
\hline \multirow{4}{*}{ A1 } & F11 & ICN & PVG & $12: 50$ & $14: 35$ \\
& F12 & PVG & MFM & $15: 40$ & $18: 20$ \\
& F13 & MFM & PVG & $19: 10$ & $21: 40$ \\
& F14 & PVG & HAN & $22: 40$ & $26: 00$ \\
\hline \multirow{4}{*}{ A2 } & F21 & PVG & CSX & $12: 10$ & $14: 00$ \\
& F22 & CSX & PVG & $14: 55$ & $16: 30$ \\
& F23 & PVG & HAK & $17: 30$ & $20: 25$ \\
& F24 & HAK & PVG & $21: 20$ & $23: 40$ \\
\hline \multirow{4}{*}{ A3 } & F31 & HRB & PVG & $13: 30$ & $16: 10$ \\
& F32 & PVG & HAK & $17: 05$ & $19: 55$ \\
& F33 & HAK & PVG & $20: 50$ & $23: 20$ \\
\hline \multirow{4}{*}{ A4 } & F41 & CGQ & PVG & $11: 40$ & $14: 00$ \\
& F42 & PVG & KWL & $14: 45$ & $17: 15$ \\
& F43 & KWL & CAN & $18: 10$ & $19: 10$ \\
& F44 & CAN & KWL & $20: 10$ & $21: 00$ \\
& F45 & KWL & PVG & $21: 55$ & $23: 55$ \\
\hline \multirow{4}{*}{ A5 } & F51 & PVG & CGQ & $13: 05$ & $15: 25$ \\
& F52 & CGQ & PVG & $16: 20$ & $18: 45$ \\
& F53 & PVG & DYG & $19: 15$ & $21: 30$ \\
& F54 & DYG & PVG & $22: 25$ & $24: 05$ \\
\hline & & PAO & PVG & $11: 00$ & $11: 55$ \\
& & PVG & CTU & $14: 55$ & $18: 20$ \\
& & & & & $21: 35$ \\
\hline & F61 & & &
\end{tabular}

TABle 2: Probability distribution of restoration time for A4.

\begin{tabular}{lccccccc}
\hline $\begin{array}{l}\text { Restoration } \\
\text { time (min) }\end{array}$ & 200 & 260 & 300 & 330 & 360 & 390 & 450 \\
\hline Probability & 0.14 & 0.15 & 0.2 & 0.15 & 0.13 & 0.12 & 0.11 \\
\hline
\end{tabular}

23 flights that operated by 6 aircraft are studied in this case. The unit delay cost $\rho_{i}$ is set to be 20 per minute, and one cancellation cost is regarded to be equivalent as 8 hours delay, which is 9,600 minutes. The cost of breaking curfew regulation $\varphi_{i}$ is 10,000 considering possible cancellation and aircraft balance. All the domestic airports have the same curfew time window [02:00, 06:00] every day; some foreign airports such as HAN do not have curfew time. A4 undergoes unexpected failure at 11:40, according to maintenance staff; its expected restoration time is 320 minutes; that is, it will be ready at 17:00. The probability distribution of the restoration time is presented in Table 2, which is obtained by statistical data from airline. The range of the restoration time varies from 200 to 450 minutes. The tests are performed on a laptop with $4 \mathrm{~GB}$ installed RAM and i5-3317U CPU $1.70 \mathrm{GHz}$.

Based on the definition in paper [23], the first stage model uses the expected values of random variables; it has model formulation $\mathrm{EV}=\min _{x_{1}} \Phi\left(x_{1}, E(\varepsilon)\right)$, where $x_{1}$ represents the decision variables in the first stage model and $\varepsilon$ represents the random variables in the recourse model. Our first stage deterministic resource assignment model is an instance of $\mathrm{EV}$. The expected value of $\mathrm{EV}$ can be represented as 


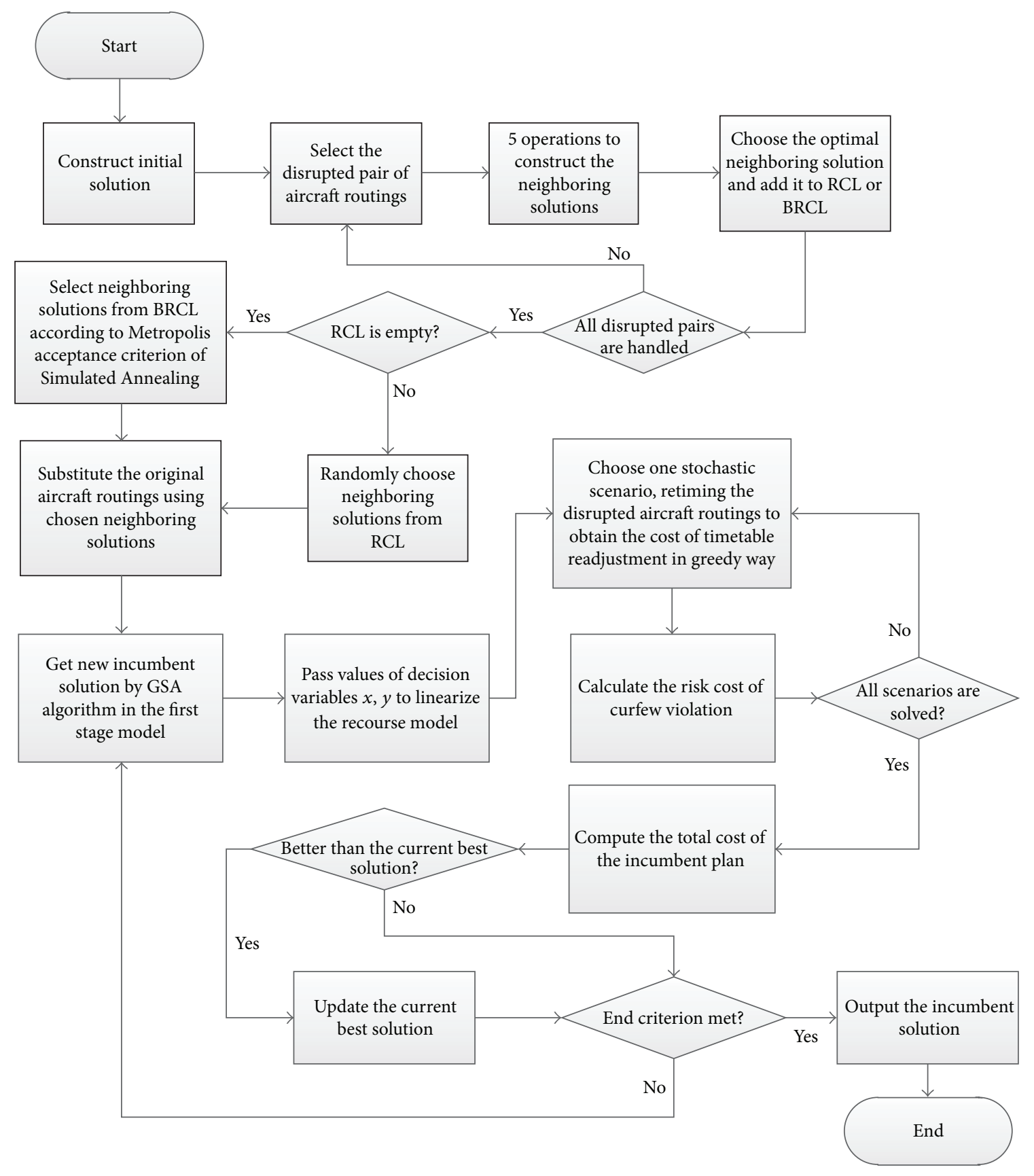

FIGURE 3: Flow chart of algorithm.

$\mathrm{EEV}=E_{\varepsilon}\left[\Phi\left(\bar{x}_{1}(\bar{\varepsilon}), \varepsilon\right)\right]$. A perfect information solution would choose optimal first period decisions for each realization of scenario. The expected value of this solution is known as "wait-and-see" (WS) solution, where WS = $E_{\varepsilon}\left[\min _{x_{1}} \Phi\left(x_{1}, \varepsilon\right)\right]$. To study the ARP in a thorough way, we solve and compare the example using models EEV, WS, and the RP proposed in Section 2, respectively.

In solving $\mathrm{EV}$, the restoration time of aircraft A4 is fixed to be 320 minutes; that is, it will be ready for use until 17:00. We run the model by GSM algorithm from Steps 1 to 6; the end criterion is set as 5 minutes of computational time or 300 incumbent solutions. Table 3 lists the final solution of the deterministic model and the total delay cost EV = $Z=15,900$. The deterministic solution will have expected recourse cost $\mathbb{Q}=5,928$; it represents the cost of adjustment due to stochasticity if deterministic solution is implemented. The stochastic expected cost of the deterministic solution will be EEV $=W=Z+\mathbb{Q}=21,828$. Similar method is used to run the WS model, where each value of probabilistic aircraft restoration time is treated as deterministic in the first stage model. After optimization and the computation on the expected value according to the probability table, we obtain 
TABLE 3: Deterministic recovery solution.

\begin{tabular}{lcc}
\hline Aircraft & Routing & Rescheduled stop over time \\
\hline A1 & F11/F62/F63/F14 & $26: 00$ \\
A2 & F21/F22/F12/F13 & $23: 00$ \\
A3 & F31/F32/F33 & $23: 20$ \\
A4 & F52/F23/F24 & $26: 00$ \\
A5 & F51/F41/F53/F54 & $24: 05$ \\
A6 & F61/F42/F43/F44/F45 & $23: 55$ \\
\hline
\end{tabular}

TABLE 4: Stochastic recovery solution.

\begin{tabular}{lcc}
\hline Aircraft & Routing & Rescheduled stop over time \\
\hline A1 & F11/F12/F13/F14 & $26: 00$ \\
A2 & F21/F22/F32/F33 & $23: 20$ \\
A3 & F31/F62/F63 & $23: 15$ \\
A4 & F52/F53/F54 & $24: 40$ \\
A5 & F51/F41/F23/F24 & $25: 00$ \\
A6 & F61/F42/F43/F44/F45 & $23: 55$ \\
\hline
\end{tabular}

WS $=16,007$. It represents the expected objective value if the decision maker can get the perfect deterministic values of random variables before optimization.

Two-stage stochastic model RP is solved by proposed stochastic algorithm; the end criterion is set as 10 minutes of computational time or 300 incumbent solutions. Table 4 shows the stochastic recovery plan result. The delay $\operatorname{cost} Z=$ 16,300 , and the cost of second stage recourse model is $\mathbb{Q}=$ 4,257; so the objective function value of the stochastic model will be $\mathrm{RP}=W=Z+\mathbb{Q}=20,557$.

As we can observe, recovery plan in Table 3 has shorter delay cost $(15,900)$ compared with stochastic plan $(16,300)$ in Table 4 without considering the uncertainty of aircraft recovery time. However, in Table 3, aircraft A4 will operate flight F24, and it will arrive at the PVG at 02:00 in the morning; there is $50 \%$ probability that F24 is cancelled or delayed a long time due to curfew breaking and will incur more cost and loss of passenger willing if that happens. That is why the stochastic recovery plan in Table 4 has lower cost $(20,557)$ compared with deterministic solution $(21,828)$ in Table 3 considering the recourse actions. In the former solution, most aircraft will land in the airports before some buffer time to the curfew time window. The changes brought by uncertainty can be absorbed; so the plan is more robust and flexible. More specifically, the expected value of perfect information (EVPI) can be defined as EVPI = RP-WS, which represents the effect of uncertainty in stochastic programs. The value of the stochastic solution VSS = EEV - RP, which is the difference between the result of using an expected value solution and the recourse problem solution. In this example, EVPI $=\mathrm{RP}-\mathrm{WS}=20,557-16,007=4,550$. The uncertainty has brought about a lot of cost to the rescheduled solution. Compare the expected scenario analysis of rescheduled plans obtained from deterministic model and the objective function value of the two-stage stochastic model; we have VSS $=\mathrm{EEV}-\mathrm{RP}=21,828-20,557=1,271$. The stochastic solution can decrease $5.8 \%$ of cost of deterministic solution, and that will be a great amount of operational cost to airlines.

\section{Conclusion}

Aircraft recovery problem due to shortage of aircraft is one of the most challenging problems in the airline operations. In this paper, the uncertainty of aircraft restoration time is introduced. The stochastic aircraft recovery problem is modeled as a two-stage stochastic recovery model. The first stage model is a deterministic resource assignment model and the second model evaluates the retiming adjustment on the solution obtained from the first stage model. Since the stochastic problem is an NP-hard problem and needs quick solution in real operation, it is impossible to use regular method to traverse the whole solution space. We designed stochastic Greedy Simulated Annealing algorithm, which combined conventional heuristic framework and simple greedy recourse method, to solve the problem. It shows the ability of obtaining satisfying solution in tractable time. A real life example is computed to analyze the proposed model and algorithm. The computational results of the stochastic model indicate the significance of considering stochastic disruption factors in the recovery problem. The study of the EVPI and VSS shows the importance of precise information and the cost-saving performance of the proposed stochastic model and algorithm.

Some interesting problems are raised for future work during the research. Besides aircraft failure, some other stochastic disruptions such as airport capacity decrease, airport temporary close, and en-route capacity change are also worthy of research. In order to increase the feasibility and accuracy of the stochastic model and algorithm in real operations, the data collection and data mining in flight irregularity should be paid more attention. Moreover, a full recovery plan for airlines consists of not only aircraft routings but also crew pairings and passenger new itineraries. Thus, research on integrated stochastic flight recovery problem is one of the interests in future work.

\section{Conflict of Interests}

The authors declare that there is no conflict of interests regarding the judgment and publication of this paper.

\section{Acknowledgments}

This work was supported by National Nature Science Foundation of China (nos. 61079014 and 71171111), funding of Outstanding Doctoral Dissertation in NUAA (no. BCXJ1314), and funding of Jiangsu Innovation Program for Graduate Education (no. CXZZ13_0174).

\section{References}

[1] D. Teodorović and S. Guberinić, "Optimal dispatching strategy on an airline network after a schedule perturbation," European Journal of Operational Research, vol. 15, no. 2, pp. 178-182, 1984. 
[2] M. F. Argüello, J. F. Bard, and G. Yu, "A GRASP for aircraft routing in response to groundings and delays," Journal of Combinatorial Optimization, vol. 1, no. 3, pp. 211-228, 1997.

[3] J. M. Rosenberger, E. L. Johnson, and G. L. Nemhauser, "Rerouting aircraft for airline recovery," Transportation Science, vol. 37, no. 4, pp. 408-421, 2003.

[4] S. Bratu and C. Barnhart, "Flight operations recovery: new approaches considering passenger recovery," Journal of Scheduling, vol. 9, no. 3, pp. 279-298, 2006.

[5] X. W. Tang, Q. Gao, and J. F. Zhu, "Research on greedy simulated annealing algorithm of irregular flight schedule recovery model," Forecasting, vol. 29, no. 1, pp. 66-70, 2010.

[6] N. Eggenberg, M. Salani, and M. Bierlaire, "Constraint-specific recovery network for solving airline recovery problems," Computers \& Operations Research, vol. 37, no. 6, pp. 1014-1026, 2010.

[7] J. D. Petersen, G. Sölveling, J.-P. Clarke, E. L. Johnson, and S. Shebalov, "An optimization approach to airline integrated recovery," Transportation Science, vol. 46, no. 4, pp. 482-500, 2012.

[8] M.-L. Le and C.-C. Wu, "Solving airlines disruption by considering aircraft and crew recovery simultaneously," Journal of Shanghai Jiaotong University (Science), vol. 18, no. 2, pp. 243252, 2013.

[9] F. T. S. Chan, S. H. Chung, J. C. L. Chow, and C. S. Wong, "An optimization approach to integrated aircraft and passenger recovery," in Proceedings of the Institute of Industrial Engineers Asian Conference, pp. 729-737, Taipei, Taiwan, July 2013.

[10] K. Sinclair, J.-F. Cordeau, and G. Laporte, "Improvements to a large neighborhood search heuristic for an integrated aircraft and passenger recovery problem," European Journal of Operational Research, vol. 233, no. 1, pp. 234-245, 2014.

[11] Y. Hu, B. Xu, J. F. Bard, H. Chi, and M. Gao, "Optimization of multi-fleet aircraft routing considering passenger transiting under airline disruption," Computers \& Industrial Engineering, vol. 80, pp. 132-144, 2015.

[12] W. Ge, J.-F. Zhu, W.-W. Wu, and X.-H. Wu, "Stochastic optimization for uncapacitated $p$-hub median problems," System Engineering-Theory \& Practice, vol. 33, no. 10, pp. 2674-2678, 2013.

[13] A. J. Schaefer, E. L. Johnson, A. J. Kleywegt, and G. L. Nemhauser, "Airline crew scheduling under uncertainty," Transportation Science, vol. 39, no. 3, pp. 340-348, 2005.

[14] J. W. Yen and J. R. Birge, "A stochastic programming approach to the airline crew scheduling problem," Transportation Science, vol. 40, no. 1, pp. 3-14, 2006.

[15] M. Dunbar, G. Froyland, and C.-L. Wu, "Robust airline schedule planning: minimizing propagated delay in an integrated routing and crewing framework," Transportation Science, vol. 46, no. 2, pp. 204-216, 2012.

[16] J. M. Rosenberger, A. J. Schaefer, D. Goldsman, E. L. Johnson, A. J. Kleywegt, and G. L. Nemhauser, "A stochastic model of airline operations," Transportation Science, vol. 36, no. 4, pp. 357-377, 2002.

[17] D. Mou and W. Zhao, "An irregular flight scheduling model and algorithm under the uncertainty theory," Journal of Applied Mathematics, vol. 2013, Article ID 361926, 8 pages, 2013.

[18] P. Arias, D. Guimarans, M. M. Mota, and G. Boosten, "A methodology combining optimization and simulation for real applications of the stochastic aircraft recovery problem," in Proceedings of the 8th EUROSIM Congress on Modelling and Simulation (EUROSIM '13), pp. 265-270, IEEE, Cardiff, Wales, September 2013.
[19] G. B. Dantzig, "Linear programming under uncertainty," Management Science, vol. 1, pp. 197-206, 1955.

[20] E. M. L. Beale, "On minimizing a convex function subject to linear inequalities," Journal of the Royal Statistical Society. Series B (Methodological), vol. 17, no. 2, pp. 173-184, 1955.

[21] J. R. Birge and F. V. Louveaux, Introduction to Stochastic Programming, Springer, Berlin, Germany, 2011.

[22] S. Y. Sohn, K. B. Yoon, and I. S. Chang, "Random effects model for the reliability management of modules of a fighter aircraft," Reliability Engineering \& System Safety, vol. 91, no. 4, pp. 433437, 2006.

[23] J. R. Birge, "The value of the stochastic solution in stochastic linear programs with fixed recourse," Mathematical Programming, vol. 24, no. 1, pp. 314-325, 1982. 


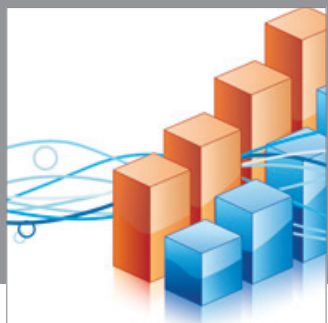

Advances in

Operations Research

mansans

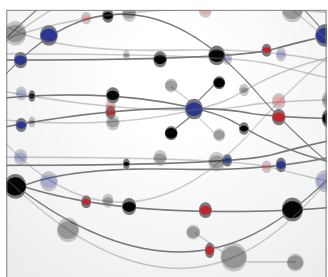

The Scientific World Journal
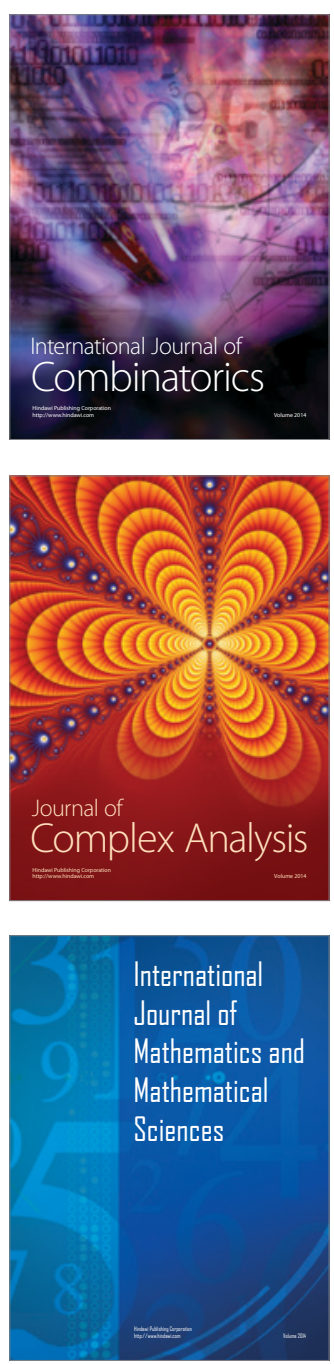
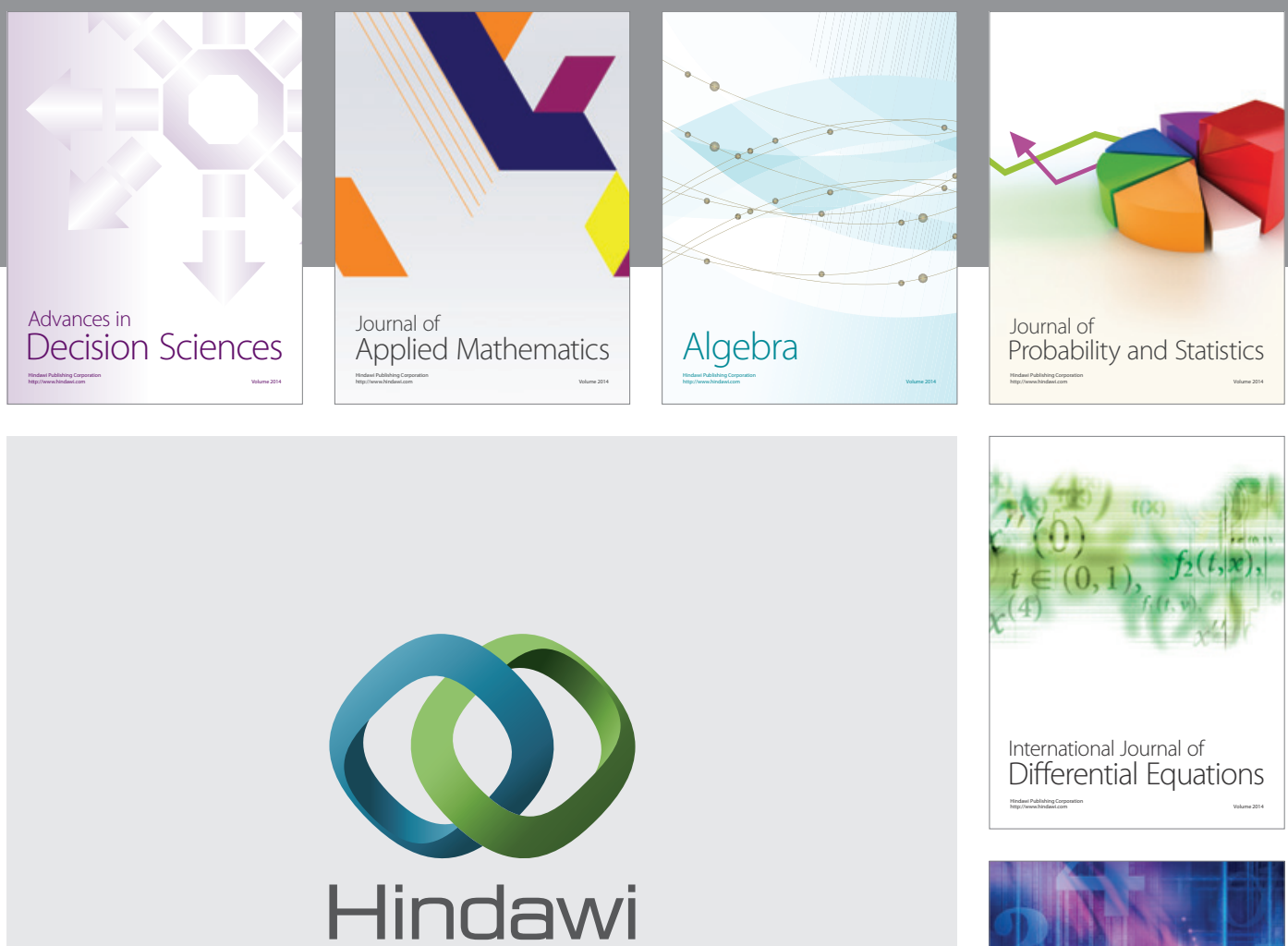

Submit your manuscripts at http://www.hindawi.com
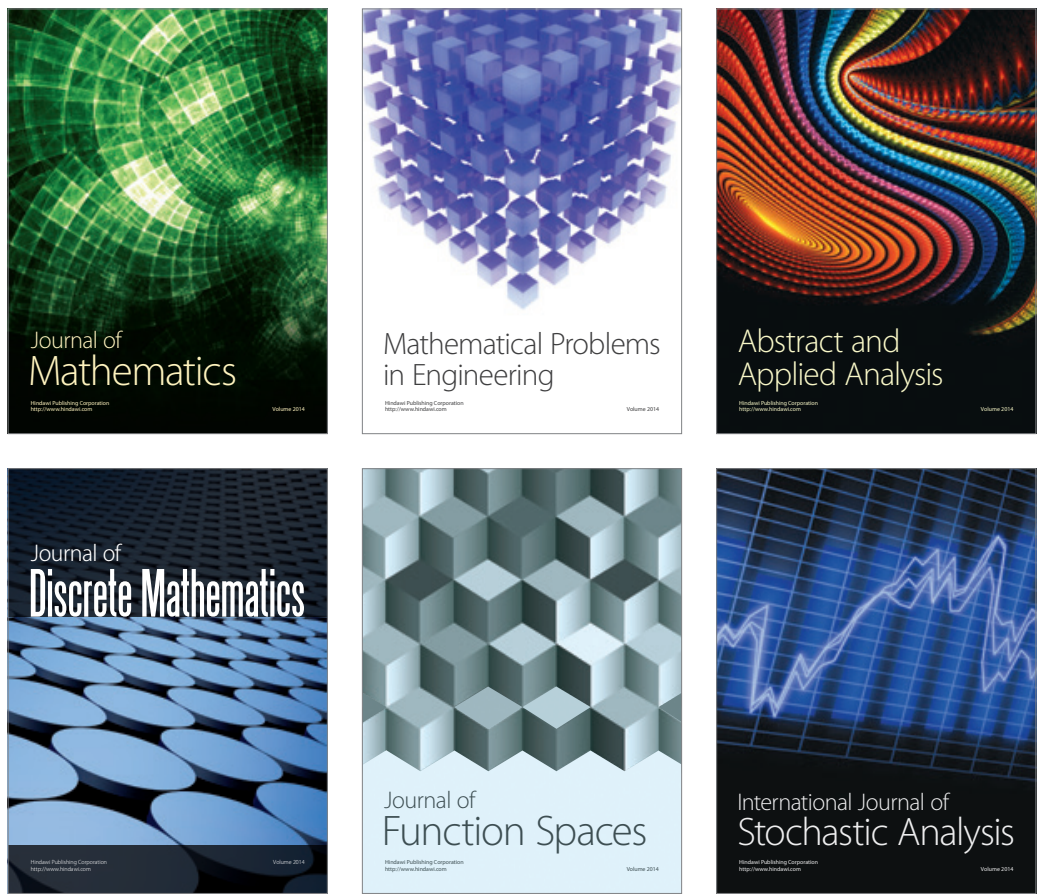

Journal of

Function Spaces

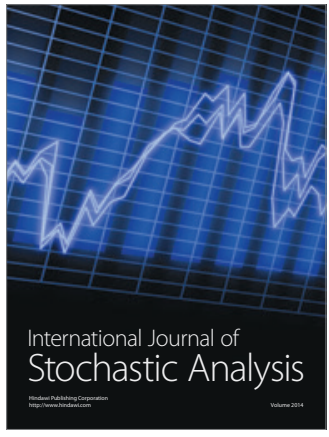

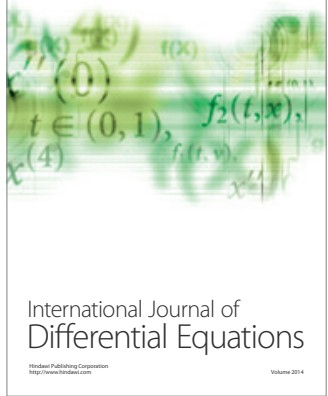
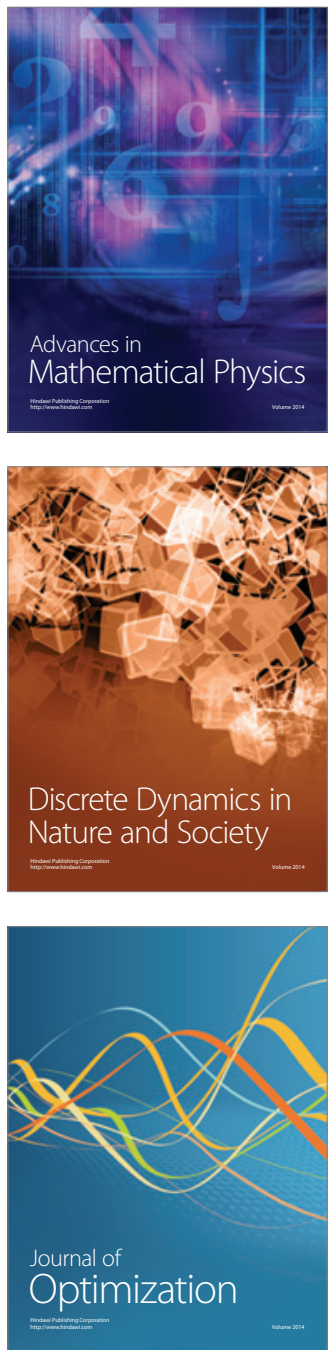